Voix et Images

voixetimages

\title{
« Transporter la mer dans ses mains »
}

\section{Paul Chamberland}

Volume 14, numéro 2 (41), hiver 1989

L’édition littéraire au Québec

URI : https://id.erudit.org/iderudit/200782ar

DOI : https://doi.org/10.7202/200782ar

Aller au sommaire du numéro

\section{Éditeur(s)}

Université du Québec à Montréal

\section{ISSN}

0318-9201 (imprimé)

1705-933X (numérique)

Découvrir la revue

\section{Citer cet article}

Chamberland, P. (1989). « Transporter la mer dans ses mains ». Voix et Images, 14(2), 336-339. https://doi.org/10.7202/200782ar d'utilisation que vous pouvez consulter en ligne.

https://apropos.erudit.org/fr/usagers/politique-dutilisation/ 
336 VOIX \& IMAGES / 41, hiver 1989

\section{«Transporter la mer dans ses mains»}

\section{par Paul Chamberland}

Un des traits dominants du journal personnel concerne la liberté dans laquelle il tient le scripteur; liberté de matière, liberté de manière. Cela fait du journal une ouvre protéiforme, aussi une auvre 
fragmentée; c'est d'ailleurs par l'accumulation de fragments diversifiés que le journal trouve lentement mais sûrement sa forme. Donc, dans la rencontre de la continuité et du morcellement. Disons dans la fragmentation continue. Ajoutons à cela que rien dans le journal n'est jamais conclu ou préalablement structuré; et que le journal prend la forme de son devenir. (p. 301-302)

- Ce passage résume de manière particulièrement nette le propos du Journal

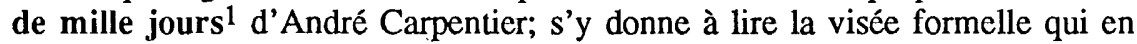
soutient l'entreprise, visée qui est elle-même indissociable de l'expérience qui conjoint écriture et vie. On y trouve désigné l'enjeu même de l'écriture, de la littérature, du faire-œuvre, qu'un autre passage propose comme «la vie dans l'écriture». De fait, le Journal donne beaucoup à penser.

Mais avant d'en venir à cette question, qui m'a paru essentielle, je veux relever d'autres aspects, dont le cadre de cette note ne me permet pas de rendre compte. L'ouvrage comporte plus de 350 pages, il s'étend, tel qu'établi en début de parcours, sur une durée de mille jours (du 26 juin 1983 au 21 mars 1986). Le projet se désignait expressément, au départ, comme celui d'un journal d'écrire ou journal d'accompagnement: à tenir en parallèle à une autre tâche d'écriture, celle d'un roman. Mais assez rapidement la direction est modifiće, le «journal d'écrire» devient un «vrai» journal, alors que le projet de roman cède la place à cclui de nouvelles. Et surtout l'écriture diaristique passe au premier plan, engageant l'attention continue du scripteur.

Assez rapidement aussi, l'ouvrage va s'élaborer conformément aux «lois» du «genre». On y trouvera donc ce qu'on trouve dans tout journal d'ćcrivain: relations d'anecdotes, rclevés d'instants ou de situations, examens d'états intérieurs; réflexions sur le travail d'ćcriture de fiction en cours (roman, nouvelles) ainsi que sur lcs catégories, problèmes qui ressortissent à ce travail; sur l'écriturc en gćnćral, la littérature, la critique, la théorie littéraire; sur l'institution et le milieu littéraires; des notes de lectures.

Je m'en suis tenu, lors de relectures attentives, aux seuls passages où l'autcur fait retour sur les dimensions et le mouvement de l'écriture diaristique en cours. Le moment du retour réflexif se renouvelle sans cesse dans le Journal. Tous les fragments de cette trame proposent, dans leur mouvance et lcur interpénétration, les éléments d'une théorie de l'écriture diaristique assez passionnante. De plus cette trame contribue à déterminer, à rythmer la forme de l'ouvrage.

J'ai donc fait un ensemble de prélèvements; je ne tiens pas compte ici de la progression en spirale qui marque leur trajectoire. Je me tiens dans l'après-coup, je fais fond sur l'cffet synthétique produit par la relecture.

La «fragmentation continue» et le libre déploiement d'une singularité, ce sont là des formules qui me paraissent mettre en relief l'enjeu de l'ouvrage.

Assez régulièrement le scripteur déplore le décousu, le morcellement, l'éparpillement aussi bien que le débordement, des morceaux en quoi s'élabore 
et se forme, de par sa nature même, le journal. Mais tout autant reconnaît-il la nécessité de la fragmentation comme modalité essentielle de l'écriture diaristique, à la fois comme textualisation et comme rapport à l'expérience, à la vie (et surtout à la relation, dialectique ou paradoxale, entre vie et écriture). Ces morceaux sont des fragments. Le terme porte déjà une longue tradition de pensée et de pratique. Depuis les premiers romantiques allemands, qui en proposaient, dans une page de l'Athenæum, la définition suivante: "petite œuvre d'art fermée comme un hérisson».

Une injonction contradictoire est donnée. Toute œuvre de fiction se propose d'elle-même, en tant qu'elle ne peut s'écrire que selon le dessein d'un achèvement formel, comme un tout, l'enjeu d'une volonté d'organisation, d'articulation serrée, de totalisation. Le journal, comme «genre», procède en revanche d'une intention inverse: en tant qu'écriture de la vie, il en suit le décousu, les multiples directions, le désordre, et de ce fait ne peut être «projeté», sans contradiction, selon une intention formelle pré-établie, totalisante. Mais du moment qu'il se hausse au premier rang dans le désir et le plaisir d'écriture, il ne peut se désigner au scripteur que comme le projet d'une œuvre, l'objet convoité d'une «construction esthétique». Du coup le scripteur est forcé d'adopter une posture paradoxalc: le seul principe formel congruent de l'écriture diaristique est celui de la fragmentation. Mais, inépuisable, inépuisée par chaque fragment, les traversant sans jamais pouvoir s'achever, la fragmentation, qui se nourrit de l'inconclu, de l'ouverture de la vie, ne peut se maintenir que si, en elle, se dérobe le "savoir absolu» d'un «système» capable de mener à un achèvement formel. L'auteur écrit, à un moment donné, qu'il a été tenté de transformer le journal en une sorte d'hybride de journal et de roman. Avec raison, me semblet-il, il a rejcté une posturc qui aurait mené à l'évacuation pure et simple de son entreprise.

Il joue le jeu, il sent, sans jamais parvenir à la formuler de manière qui le satisfasse, l'enjeu porté, mais de manière cryptique, par l'écriture de fragmentation. Il joue le jeu, cela veut dire qu'il maintient à la fois l'intotalisable et le protéiforme propre au journal et le dessein d'une élaboration, d'une mise en forme. La tension se maintiendra jusqu'à la fin, c'est-à-dire interminablement: la question restera irrésolue, mais l'inachevable aura été respecté, confirmé comme le trait formel essentiel et nécessaire à l'élaboration d'une telle «œuvre». Toutcfois, au moment de la réécriture, le principe formel parait enfin ccrnć: Un retour sur le Journal en fait la claire démonstration: il faut renoncer à toute lecture reconstructrice, retotalisante, du fragmentaire diaristique. Le journal désorganise ce qu'il organise, et d' un point de vue formel, réorganise ce qu'il désorganise. (p. 325)

«Fragmentation continue», la formule est paradoxale: la contradiction est maintenue. L'instance qui persiste à travers le discontinu des fragments c'est un «rcgard», une «conscience». Écrire un journal, des carnets, c'est, pour le scripteur, se choisir soi-même comme sujet d'énonciation. Mais la pluralité des fragments manifeste, au moment de la relecture (réécriture), une multiplicité de «je» (souvent transposés en «tu» et «il»), qui apparaissent eux-mêmes, dans «les 
lointains» du journal, comme fictifs. Une telle prolifération est elle-même doublée par une multiplicité de lecteurs (destinataires) «virtuels». Comment conjurer le vertige engendré par semblable diffraction? En maintenant la quête du sujet singulier: la fragmentation procède de «la destinée de celui qui écrit», et c'est lui qui assume la "dérive» comme "vie dans l'écriture». Chaque fragment doit se suffire à lui-même, même s'il est le «coquillage abandonné» d'un «je» en incessante métamorphose. De cette façon le mouvement de la fragmentation, qui est la «loi» de l'écriture diaristique, conjure toute tentation de «mise en forme» qui en gauchirait l'entreprise.

Je ne peux rendre compte de la finesse et de la complexité des analyses de l'auteur. Mais en terminant, je lui rendrai la parole en citant deux passages qui me paraissent suggérer fortement la perspective de l'écriture fragmentaire comme forme, son enjeu. Ce qui assure la continuité du journal personnel, sa cohérence, c'est le «je» multiple, sans doute haïssable mais rassembleur de tous les sujets, de tous les fragments et de tous les styles du journal. Tout, en effet passe par cette conscience, par ce regard. (p. 302) Dans le journal, peutêtre plus qu'ailleurs dans la littérature, la singularité, principe fondateur du «je», ressortit au style. Le style renvoie au moment de l'écriture et à ce «je» qui, écrivant, ne peut empêcher sa conscience d' imposer sa forme. (p. 303)

1 André Carpentier, Journal de mille jours [carnets 1983-1986], Montréal, Guérin littérature/XYZ éditeur, 1988, 354 p. 